Bull. Austral. Math. Soc.

$46 \mathrm{~A} 50,54 \mathrm{c} 35$

VoL. $74(2006) \quad[7-13]$

\title{
NECESSARY AND SUFFICIENT CONDITIONS FOR PRECOMPACT SETS TO BE METRISABLE
}

\author{
J.C. Ferrando, J. Kajkol and M. López Pellicer
}

This self-contained paper characterises those locally convex spaces whose (weakly) precompact (respectively, compact) subsets are metrisable. Applications and examples are provided. Our approach also applies to get Cascales-Orihuela's, Valdivia's and Robertson's metrisation theorems for (pre)compact sets.

\section{INTRODUCTION AND PRELIMINARIES}

One of the interesting problems both in topology and functional analysis deals with the question of metrisability of (precompact) compact sets. In [11] Floret (motivated by earlier results of Grothendieck, Fremlin, De Wilde and Pryce) proved an extended version of the Eberlian-Šmulian theorem with many applications, although his result said nothing about metrisability of compact subsets. Cascales and Orihuela [5] (answering a question of Floret [10]) showed that the weight of any precompact set in an $(L M)$-space is countable. Pfister and Valdivia, respectively had shown earlier the same result for $(D F)$ spaces and dual metric spaces, $[15,18]$. In [13] Kạkol and Saxon presented alternative proofs for $(L M)$-spaces and dual metric spaces. In [6] Cascales and Orihuela introduced a large class $\mathfrak{G}$ of locally convex spaces including $(L F)$-spaces and $(D F)$-spaces and presented a general result stating that:

(A) Every precompact set is metrisable in any locally convex space in class $\mathfrak{G}$.

Further progress in this direction has been achieved by Robertson [16] who used the concept of trans-separability to obtain a version of Cascales-Orihuela's result. He proved that:

(B) If a locally convex space $E$ is covered by a family $\left\{A_{\alpha}: \alpha \in \mathbb{N}^{N}\right\}$ of precompact sets which is ordered, that is $A_{\alpha} \subset A_{\beta}$ for $\alpha \leqslant \beta$, then it is trans-separable.

It is worth noting that the converse to $(B)$ fails in general. Indeed, since a locally complete Mackey space $E$ is $K$-analytic if and only if $E$ is covered by an ordered family

\section{Received 19th January, 2006}

The research of the three authors is supported by the project MTM2005 - 01182 of the Spanish Ministry of Education and Science, co-financed by the European Community (Feder projects). The research of the second author is also supported by the Komitet Badan Naukowych (State Committee for Scientific Research), Poland, grant no. 2P03A 02225 and by grant SAB2004 - 0025 from the Spanish Ministry of Education and Science

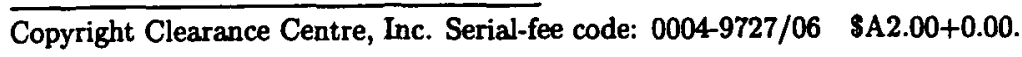


of compact sets $\left[4\right.$, Corollary 3.2] the space $\mathbb{R}^{\mathbf{R}}$ provides an elementary example of a separable Baire locally convex space which is not covered by an ordered family of precompact sets; otherwise $\mathbb{R}^{\mathbb{R}}$ would be $K$-analytic, hence metrisable by [18, 1.4 .3 (21)].

Result (B) is nicely applied in [16] to show that if the topological dual $E^{\prime}$ of a locally convex space $E$ endowed with the topology $\tau_{p}$ of uniform convergence on the precompact sets of $E$ satisfies the assumption stated in (B), every precompact set in $E$ is metrisable. Nevertheless, the converse statement fails: If $X$ is an uncountable completely regular Hausdorff $P$-space, the space $C_{p}(X)$ of all real-valued continuous functions on $X$ equipped with the pointwise topology is barrelled [3] and its dual $C_{p}(X)^{\prime}$ with the weak* topology cannot be covered by sets as stated in (B) (so the same property holds for $\left.\left(C_{p}(X)^{\prime}, \tau_{p}\right)\right)$. Indeed, otherwise $C_{p}(X)^{\prime}$ would be covered by a family $\left\{A_{\alpha}: \alpha \in \mathbb{N}^{N}\right\}$ of equicontinuous sets, and [8, Corollary 2.8] would apply to show that $X$ is countable.

Although we know from two separated sources that the statements (A) and (B) are true, we shall show that they are in fact equivalent, in the sense that one can prove (A) using (B) and, conversely, that one can prove (B) using (A). On the other hand, since there exist locally convex spaces not belonging to class $\mathfrak{G}$ for which compact sets are metrisable (this holds in $C_{p}(X)$ for compact spaces $X$ whose Souslin number is countable $[1,2]$ but $C_{p}(X) \in \mathfrak{G}$ if and only if $X$ is countable [8, Corollary 2.8]), it seems to be useful to provide necessary and sufficient conditions (which surprisingly, and very likely, have not been noticed so far) for the (pre)compact sets of a locally convex space to be metrisable.

Motivated by such situation we show first that a compact set in a completely regular Hausdorff space $X$ is metrisable if and only if $C_{c}(X)$ is trans-separable. This easily applies to prove that for a locally convex space $E$ (pre)compact sets are metrisable if and only if $E^{\prime}$ endowed with the topology of uniform convergence on the (precompact) compact sets is trans-separable (see Theorems 2 and 3 below and note that Theorem 2 also includes Valdivia's result $[18,1.4 .3(27)])$. Hence the strong dual $\left(E^{\prime}, \beta\left(E^{\prime}, E\right)\right)$ of a locally convex space $E$ is trans-separable if and only if every bounded (= precompact) set in $\left(E, \sigma\left(E, E^{\prime}\right)\right)$ is metrisable. This extends a well-known fact stating that the unit ball in a Banach space $E$ is weakly metrisable if and only if the strong dual $E^{\prime}$ is separable. Our proofs are easy, elementary and self-contained.

Finally, recall that a locally convex space $E$ belongs to class $\mathfrak{G}$ if there is a family $\left\{A_{\alpha}: \alpha \in \mathbb{N}^{N}\right\}$ of subsets of its topological dual $E^{\prime}$ (called its $\mathfrak{G}$-representation) such that:

(a) $E^{\prime}=\bigcup\left\{A_{\alpha}: \alpha \in \mathbb{N}^{N}\right\}$,

(b) $A_{\alpha} \subset A_{\beta}$ when $\alpha \leqslant \beta$,

(c) in each $A_{\alpha}$, sequences are equicontinuous,

where $\mathbb{N}$ is endowed with the discrete and $\mathbb{N}^{\mathbb{N}}$ with the product topology, respectively, [6]. Condition (c) implies that every set $A_{\alpha}$ is $\sigma\left(E^{\prime}, E\right)$-relatively countably compact. The 
class $\mathfrak{B}$ contains (among the others) $(L M)$-spaces (hence $(L F)$-spaces), the dual metric spaces (hence $(D F)$-spaces), the space of distributions $D^{\prime}(\Omega)$ and the space $A(\Omega)$ of the real analytic functions for open $\Omega \subset \mathbb{R}^{\mathbb{N}}$ (see $[8,9]$ ). By $C_{c}(X)$ we denote the space of all real-valued continuous functions on a completely regular space $X$ equipped with the compact-open topology.

\section{A CHARACTERISATION OF METRISABILITY OF (PRE)COMPACT SETS}

Recall that a locally convex space $E$ is trans-separable if for every absolutely convex neighbourhood of zero $U$ in $E$ there exists a countable subset $N$ of $E$ such that $E=N+U$, [12], see also $[14,15,16]$, where this notion has been studied (also) in uniform spaces. Every separable and every Lindelöf locally convex spaces are transseparable, and in the class of metrisable locally convex spaces the trans-separability and separability are equivalent notions. A locally convex space $E$ is trans-separable if and only if $E$ is isomorphic to a subspace of a product of separable Banach spaces. This implies in particular that every quasi-Souslin locally convex spaces $E$ (in the sense of Valdivia [18]), that is, for which there is a map $\varphi$ from $\mathbb{N}^{\mathbb{N}}$ into the family of all subsets of $E$ such that:

(i) $\cup\left\{\varphi(\alpha): \alpha \in \mathbb{N}^{N}\right\}=E$, and

(ii) if a sequence $\left\{\alpha_{n}\right\}$ in $\mathbb{N}^{\mathbb{N}}$ converges to $\alpha$ and $x_{n} \in \varphi\left(\alpha_{n}\right)$ for all $n \in \mathbb{N}$, then $\left\{x_{n}\right\}$ has an adherent point in $E$ contained in $\varphi(\alpha)$

is trans-separable and that for every locally convex space $E$ the spaces $\left(E, \sigma\left(E, E^{\prime}\right)\right)$ and $\left(E^{\prime}, \sigma\left(E^{\prime}, E\right)\right)$ are always trans-separable. The class of trans-separable locally convex spaces is stable by taking subspaces, products and completions, continuous images and projective limits. Note also, [15], that:

(C) A locally convex space $E$ is trans-separable if and only if for every neighbourhood of zero $U$ in $E$ its polar $U^{\circ}$ is $\sigma\left(E^{\prime}, E\right)$-metrisable.

Since $C_{p}(X)$ is a (dense) subspace of the product $\mathbb{R}^{X}, C_{p}(X)$ is always transseparable. For spaces $C_{c}(X)$ one has the following simple but useful

LemMa 1. The compact sets in a completely regular space $X$ are metrisable if and only if $C_{c}(X)$ is trans-separable.

Proof: If $C_{c}(X)$ is trans-separable and $K$ is a compact subset of $X$, then $C_{c}(K)$ is a separable Banach space (since the restriction map $T: C_{c}(X) \rightarrow C_{c}(K), T(f):=\left.f\right|_{K}$ is continuous and onto). Hence $K$ is metrisable. For the converse let $\left\{K_{i}: i \in I\right\}$ be the family of all compact subsets of $X$ and assume that all $K_{i}$ are metrisable. Since the map

$$
\varphi: C_{c}(X) \rightarrow \prod_{i \in I} C_{c}\left(K_{i}\right)
$$

defined by

$$
\varphi(f)=\left\{\left.f\right|_{K_{i}}: i \in I\right\}
$$


is an isomorphism onto its range and each $C_{c}\left(K_{i}\right)$ is separable, the conclusion holds.

THEOREM 2. Compact subsets of a locally convex space $E$ are metrisable if and only if $E^{\prime}$ endowed with the topology $\tau_{c}$ of uniform convergence on the compact sets of $E$ is trans-separable.

Proof: Let $F$ be the topological dual of $\left(E^{\prime}, \tau_{c}\right)$. If $\left(E^{\prime}, \tau_{c}\right)$ is trans-separable, then the $\tau_{c}$-equicontinuous subsets of $F$ are $\sigma\left(F, E^{\prime}\right)$-metrisable by (C). Since for a compact set $K$ in $E$ the bipolar $K^{\circ \circ}$ is $\tau_{c}$-equicontinuous, then $K^{\circ \circ}$ is metrisable under $\sigma\left(F, E^{\prime}\right)$. Hence $K \subseteq E$ is $\sigma\left(F, E^{\prime}\right)$-metrisable, so $\sigma\left(E, E^{\prime}\right)$-metrisable. Then $K$ is metrisable in $E$. Conversely, if all compact sets in $E$ are metrisable, by Lemma 1 the space $C_{c}(E)$ is trans-separable. As $\left(E^{\prime}, \tau_{c}\right)$ is a topological subspace of $C_{c}(E)$, the conclusion holds.

Since every quasi-Souslin locally convex space is trans-separable, our previous theorem includes Valdivia's $[18,1.4 .3(27)]$ : If $\left(E^{\prime}, \tau_{c}\right)$ is quasi-Souslin, then all compact sets in $E$ are metrisable.

THEOREM 3. Precompact sets are metrisable in a locally convex space $E$ if and only if $E^{\prime}$ endowed with the topology $\tau_{p}$ of uniform convergence on the precompact sets of $E$ is trans-separable.

Proof: If $\left(E^{\prime}, \tau_{p}\right)$ is trans-separable, by $(C)$ all precompact sets in $E$ are metrisable. Conversely, assume that all precompact sets in $E$ are metrisable. Let $\left\{P_{i}: i \in I\right\}$ be the family of all precompact sets in $E$. For every $i \in I$ let $K_{i}$ be the closure of $P_{i}$ in the completion of $E$. Since the map

$$
T:\left(E^{\prime}, \tau_{p}\right) \rightarrow \prod_{i \in I} C_{c}\left(K_{i}\right)
$$

defined by

$$
T(u)=\left\{\left.v\right|_{K_{i}}: i \in I\right\},
$$

where $v$ is the continuous linear extension of $u$ to the completion of $E$, is an isomorphism onto its range, the conclusion follows.

Since, according to (B), every locally convex space covered by an ordered family of precompact sets is trans-separable, Theorem 3 includes Robertson's [16, Corollary 2]. Likewise, Cascales-Orihuela's metrisation theorem (A) follows immediately from the previous theorem and the result (B), as the following corollary shows.

COROLlary 4. If $E$ is a locally convex space in class $\mathfrak{B}$, then $\left(E^{\prime}, \tau_{p}\right)$ is transseparable and every precompact subset of $E$ is metrisable.

Proof: Let $\left\{A_{\alpha}: \alpha \in \mathbb{N}^{N}\right\}$ be a $\mathcal{G}$-representation of $E$. Since every sequence in $A_{\alpha}$ is equicontinuous, then every $A_{\alpha}$ is $\tau_{p}$-precompact. Hence $\left(E^{\prime}, \tau_{p}\right)$ is trans-separable by virtue of $(B)$, so Theorem 3 gives the conclusion. 
Corollary 5. The strong dual $\left(E^{\prime}, \beta\left(E^{\prime}, E\right)\right)$ of a locally convex space $E$ is trans-separable if and only if every bounded set in $E$ is $\sigma\left(E, E^{\prime}\right)$-metrisable. Consequently, the strong dual of a $(D F)$-space $E$ is separable if and only if every bounded set in $E$ is weakly metrisable.

Corollaries 4 and 5 also apply to get the following well-known result.

COROLlaRY 6. (Dieudonné) Every Fréchet-Montel space $E$ is separable.

Proof: Since $\left(E^{\prime}, \beta\left(E^{\prime}, E\right)\right)$ is a Montel $(D F)$-space, we apply Corollary 4 to show that every $\sigma\left(E^{\prime}, E\right)$-bounded set is metrisable. Then the reflexivity of $E$ and Corollary 5 show that $E$ is separable.

Note the following link between the arguments presented by Cascales-Orihuela and Robertson, respectively.

PROPOSITION 7. The statements $(A)$ and $(B)$ are equivalent.

Proof: $(\mathrm{B}) \Rightarrow(\mathrm{A})$ follows from Corollary 4 . (A) $\Rightarrow(\mathrm{B})$ : Let $E$ be a locally convex space covered by an ordered family $\left\{A_{\alpha}: \alpha \in \mathbb{N}^{N}\right\}$ of precompact sets. We may assume that the sets $A_{\alpha}$ are absolutely convex. Let $\tau_{\sigma}$ be a locally convex topology on $E^{\prime}$ whose basis of neighbourhoods of zero is composed by sets

$$
t\left(A_{\alpha}\right)^{\circ}, \quad 0<t \leqslant 1, \quad \alpha \in \mathbb{N}^{\mathbb{N}} .
$$

Then $F:=\left(E^{\prime}, \tau_{\varpi}\right)$ belongs to class $\mathfrak{G}$. Indeed, for every $\alpha=\left(n_{k}\right) \in \mathbb{N}^{\mathbb{N}}$ set $K_{\alpha}:=n_{1}\left(A_{\alpha}^{\circ}\right)^{\circ}$, where the last $\circ$ is the polar in $\left(F^{\prime}, \sigma\left(F^{\prime}, F\right)\right)$. Then $\left\{K_{\alpha}: \alpha \in \mathbb{N}^{N}\right\}$ is a $\mathfrak{G}$-representation of $F$. To show that $E$ is trans-separable we need only to prove that the polar $U^{\circ}$ of every neighbourhood of zero $U$ in $E$ is $\sigma\left(E^{\prime}, E\right)$-metrisable. This is clear since

$$
\left.\sigma\left(E^{\prime}, E\right)\right|_{U^{\circ}}=\left.\tau_{p}\right|_{U^{\circ}}=\left.\tau_{\odot}\right|_{U^{\circ}}
$$

and $U^{\circ}$ is $\tau_{\odot}$-metrisable (by (A)).

Corollary 4 may suggest the following question: Is it true that a locally convex space $E$ for which $E^{\prime}$ is covered by an ordered family $\left\{A_{\alpha}: \alpha \in \mathbb{N}^{\mathbb{N}}\right\}$ of $\tau_{p}$-precompact sets necessarily belongs to class $\mathfrak{B}$ ? Example 8 answers this question in the negative.

EXAMPLE 8. Let $E$ be an infinite-dimensional separable reflexive Banach space. Since $\left(E^{\prime}, \beta\left(E^{\prime}, E\right)\right)$ is a $K$-analytic Banach space, $E^{\prime}$ is covered by an ordered family $\left\{K_{\alpha}\right.$ : $\left.\alpha \in \mathbb{N}^{\mathbb{N}}\right\}$ of $\beta\left(E^{\prime}, E\right)$-compact sets, [17]. Clearly $\beta\left(E^{\prime}, E\right)$ is the topology $\tau_{p}$ of uniform convergence on the $\sigma\left(E, E^{\prime}\right)$-bounded sets. But $E_{\sigma}:=\left(E, \sigma\left(E, E^{\prime}\right)\right)$ does not belong to class $\mathfrak{G}$. Otherwise, since $E_{\sigma}$ is dense in the product $\mathbb{R}^{I}$ for some set $I$, the space $\mathbb{R}^{I}$ would also belong to class $\mathfrak{G}$, [6, Proposition 8]. But $\mathbf{R}^{I}$ is a Baire space, so [7, Theorem 4.8] applies to show that $\mathbf{R}^{I}$ has countable tightness, that is, for every $A \subset \mathbb{R}^{I}$ and every $x \in \bar{A}$ there exists a countable set $B \subseteq A$ such that $x \in \bar{B}$. Consequently, $I$ is countable and $E$ is finite-dimensional, a contradiction. 
In [6, Corollary 1.15] Cascales and Orihuela showed that for a separable locally convex space $E$ in class $\mathfrak{G}$ its dual $\left(E^{\prime}, \tau_{p}\right)$ is even analytic (hence separable). This result fails however if we drop the assumption on $E$ to be in class $\mathfrak{B}$.

EXAMPLE 9. Let $F$ be a one-codimensional dense linear subspace of $\ell_{1}$ which is a Baire space [18, 1.1.3 (6)]. Note that $F$ is not quasi-Souslin; otherwise $F$ would be complete by $[18,1.4 .3(21)]$. Put $E:=\left(c_{0}, \sigma\left(c_{0}, F\right)\right)$. Since $\sigma\left(c_{0}, F\right) \leqslant \sigma\left(c_{0}, \ell_{1}\right)$, then $E$ is separable. On the other hand, since $F$ is barrelled and dense in $\ell_{1}$, then

$$
\left.\beta\left(\ell_{1}, c_{0}\right)\right|_{F}=\beta\left(F, \ell_{\infty}\right)
$$

Hence

$$
\beta\left(F, c_{0}\right) \leqslant \beta\left(F, \ell_{\infty}\right)=\left.\beta\left(\ell_{1}, c_{0}\right)\right|_{F} \leqslant \beta\left(F, c_{0}\right)
$$

and

$$
\beta\left(F, c_{0}\right)=\left.\beta\left(\ell_{1}, c_{0}\right)\right|_{F} .
$$

Consequently, the topology $\tau_{p}$ on $F$, that is $\beta\left(F, c_{0}\right)$, coincides with the norm topology $\left.\beta\left(\ell_{1}, c_{0}\right)\right|_{F}$ of $F$. Therefore $E$ is a separable locally convex space not belonging to class $\mathfrak{G}$ whose dual $\left(F, \tau_{p}\right)$ is not analytic.

\section{REFERENCES}

[1] A.V. Arkhangel'skii, Topological function spaces, Mathematics and its Applications (Soviet Series) 78 (Kluwer Academic Publishers Group, Dordrecht, 1992).

[2] A.V. Arkhangel'skii, General Topology III (Springer-Verlag, Berlin, Heidelberg, New York, 1995).

[3] H. Buchwalter and J. Schmets, 'Sur quelques propiétés de l'espace $C_{s}(T)^{\prime}$, J. Math. Pures Appl. 52 (1973), 337-352.

[4] B. Cascales, 'On $K$-analytic locally convex spaces', Arch. Math. (Basel) 49 (1987), 232-244.

[5] B. Cascales and J. Orihuela, 'Metrizability of precompact subsets in ( $L F)$-spaces', Proc. Edinburgh Math. Soc. 103 (1986), 293-299.

[6] B. Cascales and J. Orihuela, 'On compactness in locally convex spaces', Math. $Z .195$ (1987), 365-381.

[7] B. Cascales, J. Kgkol and S.A. Saxon, 'Weight of precompact sets and tightness', J. Math. Anal. Appl. 269 (2002), 500-518.

[8] B. Cascales, J. Kakol and S.A. Saxon, 'Metrizability vs. Fréchet-Urysohn property', Proc. Amer. Math. Soc. 131 (2003), 3623-3631.

[9] J.C. Ferrando, J. Kakol, M. López Pellicer and S.A. Saxon, 'Tightness and distinguished Fréchet spaces', J. Math. Anal. Appl. (to appear).

[10] K. Floret, 'Some aspects of the theory of locally convex inductive limits', in Functional Analysis: Surveys and Recent Results, II, Proc. Conf. Functional Anal. Univ. Padeborn, Padeborn 1979 (North-Holland, Amsterdam, 1980), pp. 205-237.

[11] K. Floret, Weakly compact sets, Lecture Notes in Math. 801 (Springer-Verlag, Berlin, 1980). 
[12] D. Gullick and J. Schmets, 'Separability and semi-norm separability for spaces of bounded continuous functions', Bull. Roy. Sci. Liége 41 (1972), 254-260.

[13] J. Kakol and S.A. Saxon, 'Montel $(D F)$-spaces, sequential $(L M)$-spaces and the strongest locally topology', J. London Math. Soc. 66 (2002), 388-406.

[14] H.P.A. Künzi, M. Mršević, I.L. Reilly and M.K. Vamanamurthy, 'Pre-Lindelöf quasi-pseudo-metric and quasi-uniform spaces', Mat. Vesnik. 46 (1994), 81-87.

[15] H.H. Pfister, 'Bemerkungen zum Satz über die separabilität der Fréchet-Montel Raüme', Arch. Math. (Basel) 27 (1976), 86-92.

[16] N. Robertson, 'The metrisability of precompact sets', Bull. Austral. Math. Soc. 43 (1991), 131-135.

[17] M. Talagrand, 'Espaces de Banach faiblement $K$-analytiques', Ann. Math. 110 (1979), 407-438.

[18] M. Valdivia, Topics in locally convex spaces, Notas de Matemática 85 (North Holland, Amsterdam, 1982).

Centro de Investigación Operativa

Universidad Miguel Hernández

E-03202 Elche (Alicante)

Spain

e-mail: jc.ferrando@umh.es
Faculty of Mathematics and Informatics

A. Mickiewicz University

61-614 Poznań

Poland

e-mail: kakol@amu.edu.pl

Departamento de Matemática Aplicada and IMPA

Universidad Politécnica

E-46022 Valencia

Spain

e-mail: mlopezpe@mat.upv.es 